\title{
Trigeminal Ganglion
}

National Cancer Institute

\section{Source}

National Cancer Institute. Trigeminal Ganglion. NCI Thesaurus. Code C62642.

Large sensory gang lion of the trigeminal nerve that lies adjacent to the cavernous sinus

in the trigeminal cavity of the dura mater. 